\title{
Orientational Imaging of Subwavelength Au Particles with Higher Order Laser Modes
}

2006

Vol. 6, No. 7

1374-1378

\author{
Antonio Virgilio Failla, ${ }^{\dagger}$ Hui Qian, ${ }^{\dagger}$ Huihong Qian, Achim Hartschuh, and \\ Alfred J. Meixner* \\ Institute of Physical and Theoretical Chemistry, University of Tuebingen, \\ Auf der Morgenstelle 8, D-72076 Tuebingen, Germany
}

Received February 14, 2006; Revised Manuscript Received May 31, 2006

\begin{abstract}
We present a new method for the imaging of single metallic nanoparticles that provides information about their shape and orientation. Using confocal microscopy in combination with higher order laser modes, scattering images of individual particles are recorded. Gold nanospheres and nonorods render characteristic patterns reflecting the different particle geometries. In the case of nanorods, the scattering patterns also reveal the orientation of the particles. This novel technique provides a promising tool for the visualization of nonbleaching labels in the biosciences.
\end{abstract}

The optical properties of gold nanoparticles are influenced strongly by particle plasmons (PPs), that is, the collective oscillations of free electrons in the conduction band. A large number of theoretical and experimental studies were dedicated to the understanding of PP physics and revealed fascinating properties. ${ }^{1-4}$ For example, PPs in gold particles are linked to broad band photoluminescence ${ }^{2}$ and strong local field enhancement, making these nanostructures promising candidates for a variety of different applications. ${ }^{4}$

In addition, the high biocompatibility of gold nanoparticles has stimulated their widespread use in the biosciences within the last few years. ${ }^{5-10}$ Gold particles were used as intramolecular distance rulers, ${ }^{5}$ as non bleaching sensors, ${ }^{6}$ and as labels for multiphoton microscopy. ${ }^{10}$ Simultaneously, novel optical microscopic techniques were developed in order to detect and characterize single nanoparticles. ${ }^{11-14}$ Confocal dark-field microscopy was used in conjunction with polarization-sensitive detection to determine the relative orientation of $\mathrm{Au}$ nanorods ${ }^{11}$ (i.e., the magnitudes of the projections of the long axis of a nanorod onto perpendicular polarization axis). Furthermore, by controlling the orientation of an $\mathrm{Au}$ particle attached on a sharp glass fiber tip and excitation by a white light beam and by measuring the scattering intensity and spectra as a function of the incident polarization it was possible to obtain information about the particle orientation and degree of elipsticity. ${ }^{13}$ In another study, the light scattered by individual gold nanoparticles was collected using a sharp optical fiber tip. ${ }^{14}$ However, for isolated particles distributed

\footnotetext{
* Corresponding author. E-mail: alfred.meixner@ipc.uni-tuebingen.de.

$\dagger$ These authors contributed equally to this study.
}

on a flat surface with random orientation none of these methods can reveal information of their shape and, in the case of elongated particles, determine the absolute orientation at the same time.

Gold nanospheres were purchased from Sigma-Aldrich, with a diameter of $20 \pm 3 \mathrm{~nm}$ as specified by the manufacturer. Gold nanorods were produced by a seedmediated method described by El-Sayed et al. ${ }^{15}$ All reagents were obtained from Sigma-Aldrich and used as received. Ultrapure deionized water was used in all of the experiments. The samples were prepared by spin-coating diluted suspensions of gold nanospheres and gold nanorods onto cleaned microscope cover slides. The distribution and the size of the nanoparticles were determined by atomic force microscopy (AFM) with a Digital Instruments Nanoscope IIIA in tapping mode using standard silicon nitride tips. Extinction spectra were recorded on a Lambda $9 \mathrm{UV} / \mathrm{vis}$ spectrophotometer (Perkin-Elmer).

For an initial sample characterization, the average size of the nanoparticles was determined from their extinction spectra in solution (Figure 1a and b) and from AFM measurements (Figure 1c and d). Gold nanospheres and gold nanorods exhibit different characteristic plasmonic properties because of their different shapes. ${ }^{4}$ Although nanospheres have only one plasmon band (see Figure 1a), gold nanorods feature two plasmon bands: the first is determined by the short axis, and the second is related to the long axis (see Figure 1b). ${ }^{15,16}$ Practically, the extinction spectra can be fitted by a theoretical simulation based on the extension of Mie theory. ${ }^{17}$ The measurements indicated that the diameter of the gold 


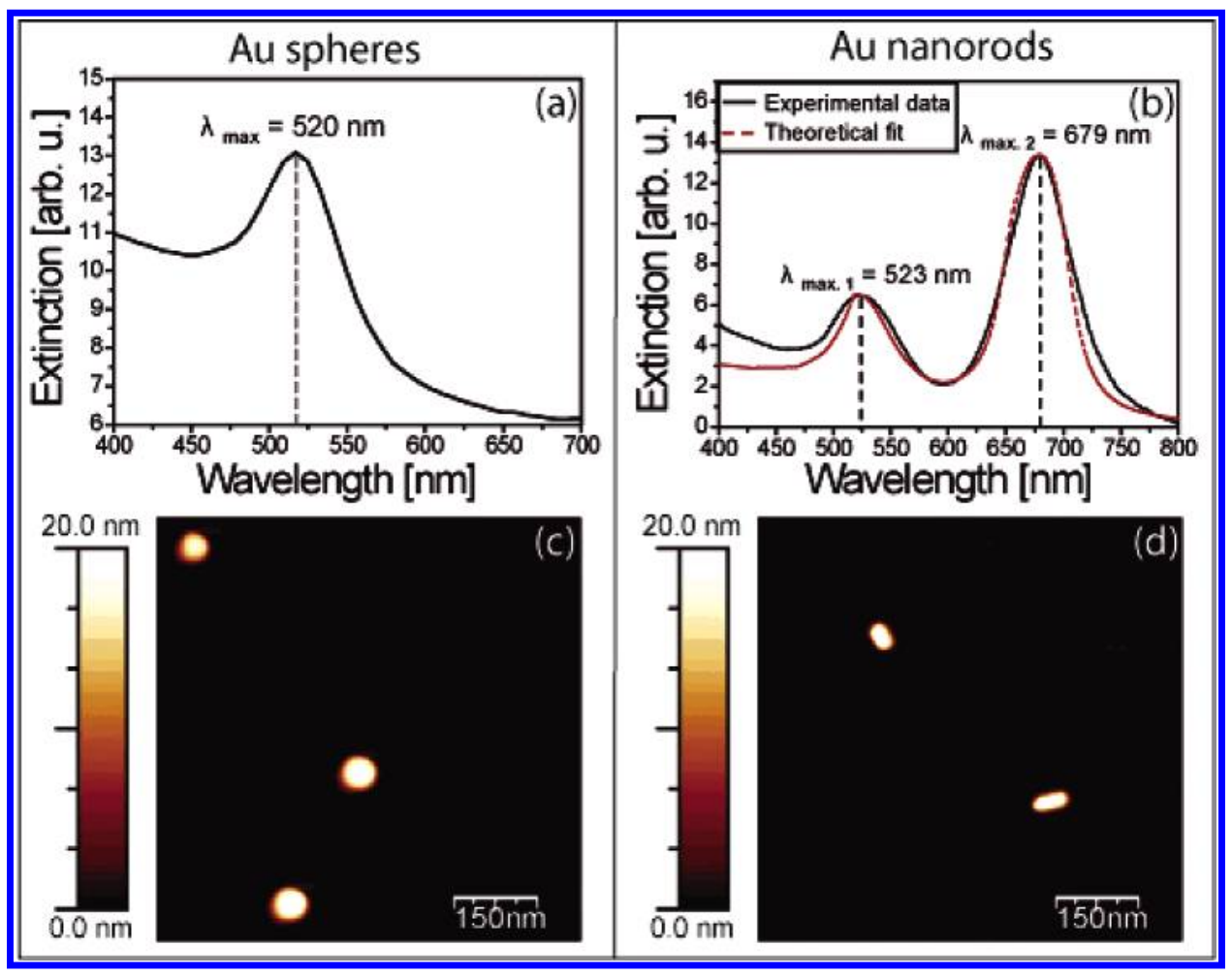

Figure 1. (a) UV-vis excitation spectrum of an ensemble of Au nanospheres and (c) AFM images of the respective individual gold nanospheres with a nominal diameter of $20 \mathrm{~nm}$ as specified by the manufacturer. (b) Experimental (black full line) and calculated (dotted red line) extinction spectra of Au nanorods (12-15 $\mathrm{nm}$ in diameter and aspect ratio of about 2.4) and respective AFM image (d) showing individual particles.

nanospheres is about $20 \pm 3 \mathrm{~nm}$ in agreement with the manufacturer specification. The aspect ratio of the gold nanorods studied in this work was found to be about $2.4 \pm$ 0.1 with widths between 12 and $15 \mathrm{~nm}$ and lengths between 30 and $45 \mathrm{~nm}$.

All single-particle optical studies were performed with a home-built inverted confocal microscope similar to that in ref 18 . A linearly polarized $\mathrm{He}-\mathrm{Ne}$ laser $(\lambda=632.8 \mathrm{~nm})$ served as the excitation light source. Either the fundamental Gaussian laser mode or higher order doughnut modes were used. In this work, azimuthally and radially polarized doughnut modes are generated by a polarization converter with four half-wave plates in each quadrant, similar to that used by Leuchs et al. ${ }^{19}$ In Figure 2a, the synthesis of radially and azimuthally polarized doughnut modes is described schematically. A spatial filter was used to remove higher spatial frequencies. Figure $2 b$ shows a photograph of the beam profile while the calculated beam profiles of azimuthally and radially polarized doughnut modes in the focus of a high NA objective are shown in Figure $2 \mathrm{c}$ and $\mathrm{d}$, respectively. The collimated beam was reflected by a nonpolarizing 50:50 beam splitter and focused by an oil immersion microscope objective $($ N.A. $=1.25)$ onto the sample surface. Both the scattered light from the single particles and the reflected light from the glass object slide were collected with the same objective, transmitted by the beam splitter and detected by a photon-counting avalanche photodiode (APD).
The signal in our configuration results from the superposition of the laser field reflected at the interface $\boldsymbol{E}_{\mathrm{r}}$ and the field scattered by the nanoparticle $\boldsymbol{E}_{\mathrm{s}}$ :

$$
I_{\text {det }} \propto\left|E_{\mathrm{r}}+E_{\mathrm{s}}\right|^{2}=\left|E_{\mathrm{r}}\right|^{2}+\left|E_{\mathrm{s}}\right|^{2}+2\left|E_{\mathrm{r}}\right|\left|E_{\mathrm{s}}\right| \cos (\gamma)
$$

Three terms appear on the right-hand side of eq 1 . The first term, proportional to the square of the reflected field, represents the background intensity. The second term indicates the pure scattering signal. The interference term is determined by both fields and their phase relation. For nanoparticles, the amplitude of the scattered field $\left|\boldsymbol{E}_{\mathrm{s}}\right|$ is proportional to the third power of the average particle diameter and will thus be much smaller than the amplitude of the field reflected at the interface $\left|\boldsymbol{E}_{\mathrm{r}}\right|$. This is clearly the case for our configuration where light is focused on a glass/ air interface using a high numerical aperture objective leading to total internal reflection for light waves coming in at angles that are larger than the critical angle $\theta_{\mathrm{c}}=a \sin \left(1 / n_{\text {glass }}\right) \approx$ $42^{\circ}$. Hence, the pure scattering signal $\left|\boldsymbol{E}_{\mathrm{s}}\right|^{2}$ can be neglected compared to the signal caused by reflection $\left|\boldsymbol{E}_{\mathrm{r}}\right|^{2}$. The interference term, however, is scaled by the strength of the reflected field, giving rise to a detectable signal even from single particles. The sign of this signal and the image contrast depends sensitively on the phase relation, $\gamma$, that is determined by the plasmon resonance of the particle and by phase terms resulting from total internal reflection. 


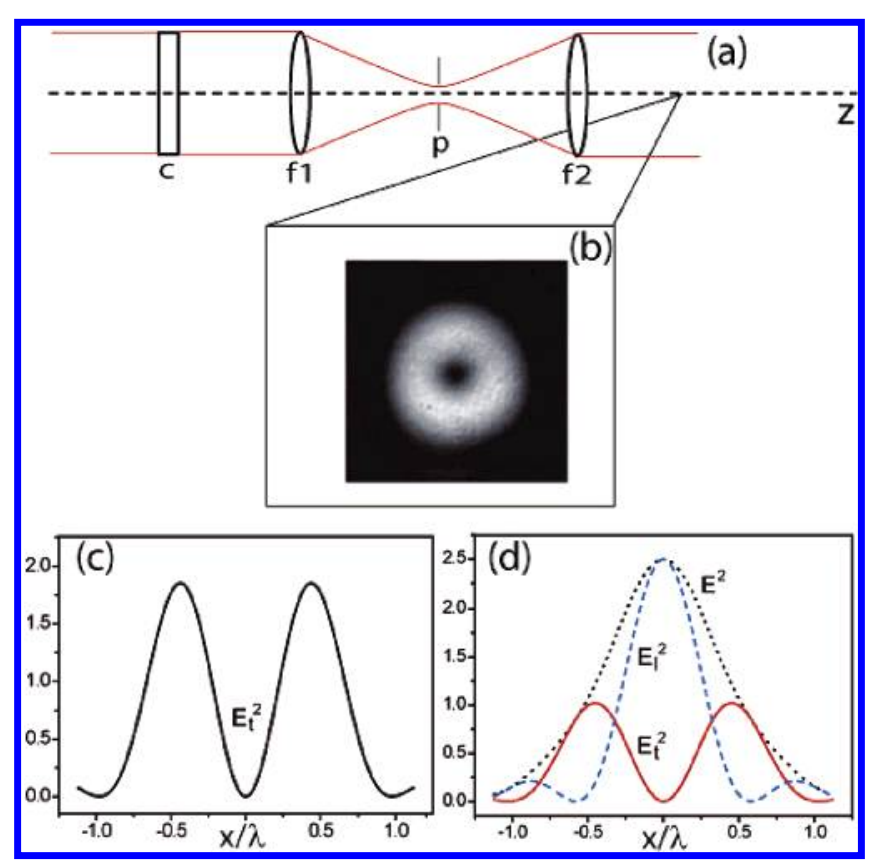

Figure 2. (a) Synthesis of an azimuthally/radially polarized doughnut mode from a collimated linearly polarized Gaussian beam. In short a mode converter plate, $\mathrm{C}$, with four quadrants consisting of $\lambda / 2$ plates, which are rotated $90^{\circ}$ with respect to each other, divides the beam in four parts with opposite polarizations as described in ref 19 . These four components are superimposed by a spatial filter consisting of two lenses, $f_{1}$ and $f_{2}$, and a pinhole, $p$, giving rise to azimuthally/radially polarized doughnut mode whit a profile shown in the photograph in Figure $2 \mathrm{~b}$. The spatial filter was used to cut off higher order spatial frequencies. (c and d) Calculated beam profiles of azimuthally and radially polarized doughnut modes in the focus of a high numerical aperture objective $(\mathrm{NA}=1.25) . E_{\mathrm{t}}^{2}$ is the transversal, $E_{1}^{2}$ is the longitudinal (parallel to the optical axis of the microscope), and $E^{2}$ is the total field strength. Note that for azimuthally polarized doughnut modes $E^{2}$ $=E_{\mathrm{t}}^{2}$.

A quantitative description of both fields and their phase relation can be obtained analytically using the field distribution of the laser mode, the numerical aperture of the focusing lens, the Fresnel coefficients of the glass/air interface, and the polarizability tensor of the particle. Details of this procedure, that is, based on work in refs 20,21 , will be published elsewhere. Here, we only show the numerical results adapted to our experimental situation to illustrate the feasibility of such calculations and to further support our experimental findings.

Scattering images generated by raster scanning a gold sphere and a gold nanorod through a tightly focused linearly polarized Gaussian beam are shown in Figure 3a and b. Both images show a single dark spot corresponding to lower intensity and it is obviously impossible to distinguish nanospheres and nanorods by their scattering patterns. The reduced intensity indicates destructive interference between the light scattered by the particles and the light reflected from the glass/air interface as described in eq 1. In Figure $3 c-f$, the same particles are imaged using azimuthally (Figure 3c and d) and radially polarized doughnut modes (Figure $3 \mathrm{e}$ and f). The patterns rendered by the sphere show almost perfect radial symmetry in all cases, as expected from the

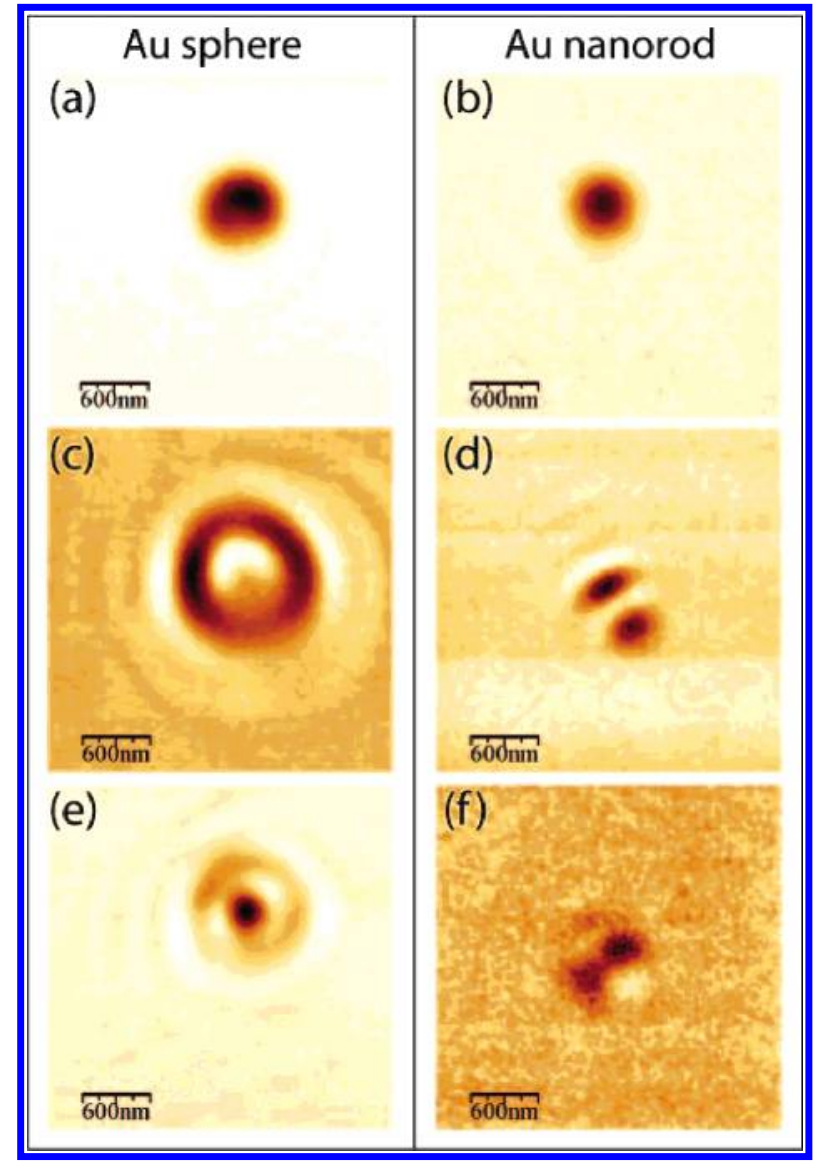

Figure 3. Scattering images of the same spherical gold nanoparticle (left) (nominal diameter $20 \mathrm{~nm}$ ) and the same gold nanorod (right) (nominal aspect ratio 2.4, and minor axis diameter 15/20 nm) using different laser modes: ( $a$ and b) for a linearly polarized Gaussian beam; (c and d) for an azimuthally polarized doughnut mode; and (e and f) for a radially polarized doughnut mode. Note that the sphere and the nanorod can be distinguished by their scattering patterns only in the case of doughnut modes.

isotropic polarizability of the particle and the radial symmetry of the intensity profile of the laser modes. The patterns rendered by the nanorod, however, are clearly different and reflect its nonisotropic polarizability. Consequently, from a single scattering pattern particles with isotropic and anisotropic polarizability can be distinguished.

To establish a correlation between the orientation of the nanorods and the resulting scattering patterns, we performed AFM measurements of the nanoparticles. In Figure 4, topography and both experimental and theoretical scattering images of two individual nanorods with different orientations are presented. All patterns show a double-lobe structure that is rotated by $90^{\circ}$ when changing from the azimuthally to the radially polarized mode. By comparing the scattering patterns with the focal fields of the laser modes shown schematically in Figure 2, it can be seen that the strongest signal contribution results from field components in the direction of the long axis of the nanorod. The plasmon resonance of the main axis of the nanorod is at least 1 order of magnitude stronger than the one related to the short axis at this wavelength, as determined from the extinction spectra in Figure 1, in agreement with ref 4 . In other words, these 


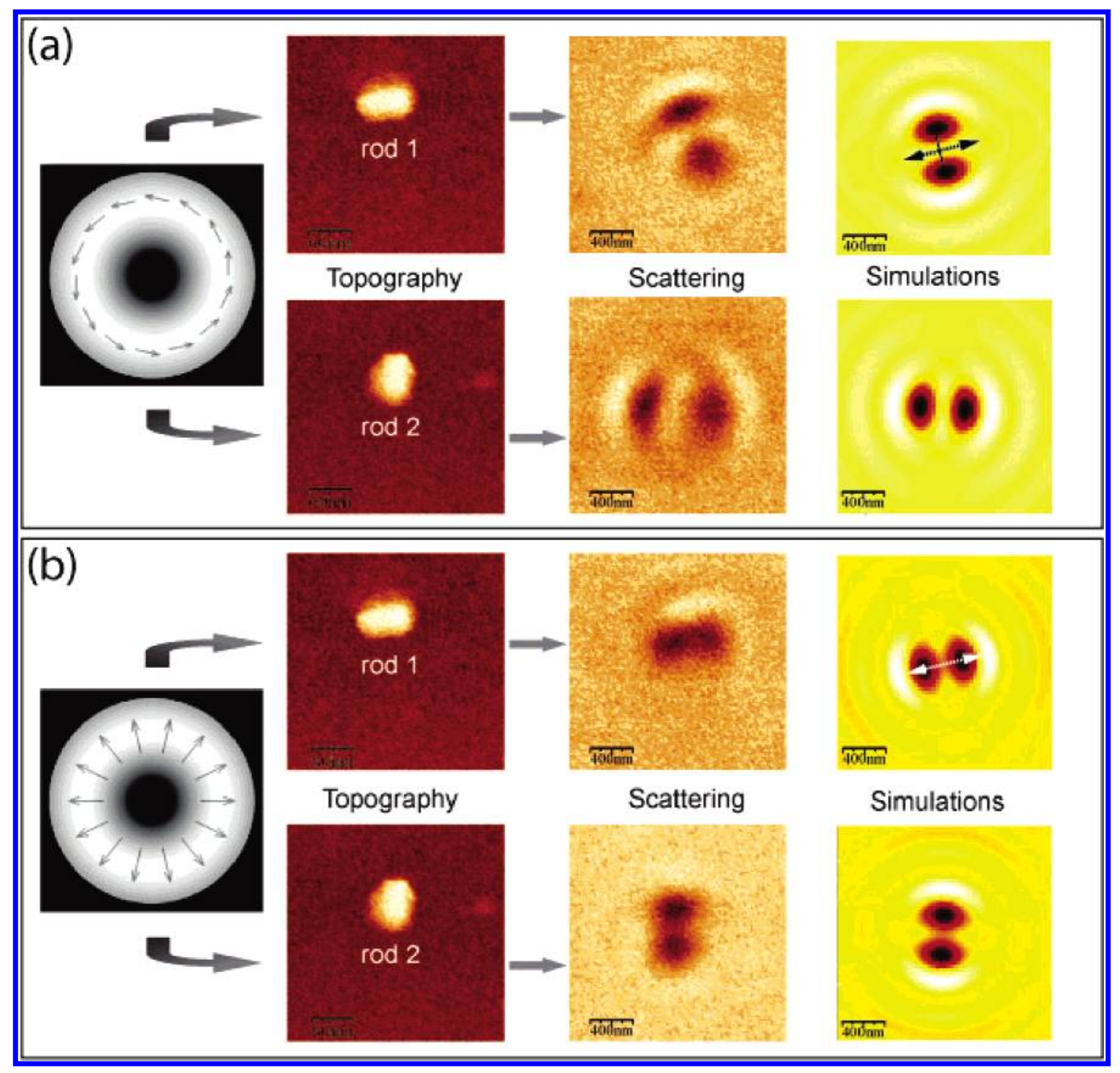

Figure 4. Topography and scattering images of two gold nanorods (rod 1,2 as marked) with different orientations. The scattering images are generated by azimuthally and radially polarized doughnut modes, a and b, respectively. The panels on the left show schematically the respective field distributions of the transverse components of the two laser modes, see also Figure 2 . In the right column in a and $b$ the simulated pattern of the same rods are depicted. Note that the orientation of the nanorods can be determined from the scattering images (see arrows).

patterns reflect the orientation of the tensor of anisotropic scatterers. Hence, in the case of the radial mode (azimuthal mode) the line connecting the two lobes directly indicates the implane orientation of the long (short) axis of the nanorod. From our optical, AFM, and simulation data, the orientation of gold nanorods can be determined with an accuracy better than $5^{\circ}$.

In general, the orientation of nanorods, that is, their projection on a plane perpendicular to the direction of light propagation, can also be partially obtained by using linearly polarized laser-light ${ }^{24}$ or incoherent white light ${ }^{11}$ but at the cost of a more complicated experimental setup and acquisition processes. In fact, the virtue of our method is to visualize the particle orientation directly by one single image. The method presented here also has the potential to determine the orientation in three dimensions in analogy to the detection of the orientation of single fluorescent molecules described in refs 22 and 23 . For this purpose, a radially polarized beam would be used that provides electric field components both in longitudinal and transverse directions depending on the position with respect to the focal center (see Figure 2d). For gold nanorods with arbitrary 3D orientation, the two lobed patterns observed for those particles oriented along the sample surface will change gradually into a circular pattern for those with their long axis perpendicular to the surface, which will be shown in another publication.

In conclusion, a new method for observing individual gold nanoparticles was developed. Our approach is based on farfield confocal microscopy in combination with higher order laser modes and allows one to detect and characterize subwavelength-sized metallic particles in a simple but effective way. By observing the characteristic scattering patterns of gold nanoparticles, one can clearly distinguish the shape of nanospheres and nanorods and directly determine the orientation of nanorods. In summary the possibility for distinguishing and determining the shape of single $\lambda / 15$ 
sized metal particles by tightly focused higher order laser beams can be considered as an important progress of confocal microscopy.

Acknowledgment. We acknowledge stimulating discussions with Mathias Steiner and Dr. Catrinel Stanciu. We gratefully acknowledge financial support by DFG (Me 1600/ 6-1/2).

\section{References}

(1) Bouhelier, A.; Beversluis, M. R.; Novotny, L. Appl. Phvs. Lett. 2003, $83,5041-5043$.

(2) Beversluis, M. R.; Bouhelier, A.; Novotny, L. Phys. Rev. B 2003, 68, 1154331-11543310.

(3) Klar, T.; Perner, M.; Grosse, S.; von Plessen, G.; Spirkl, W.; Feldmann, J. Phvs. Rev. Lett. 1998, 80, 19 4249-4252.

(4) Sönnichsen, C.; Franzl, T.; Wilk, T.; von Plessen, G.; Feldmann, J. Phvs. Rev. Lett. 2002, 88, 077402-1-077402-4.

(5) Reinhard, B. M.; Siu, M.; Agarwal, H.; Alivisatos, A. P.; Liphardt, J. Nano Lett. 2005, 5, 2246-2252.

(6) Raschke, G.; Kowarik, S.; Franzl, T.; Sönnichsen, C.; Klar, T. A.; Feldmann, J. Nano Lett. 2003, 3, 935-938.

(7) Bauer, L. A.; Birenbaum, N. S.; Meyer, G. J. J. Mater. Chem. 2004, $14,517-526$

(8) El-Sayed, I. H.; Huang, X.; El-Sayed, M. A. Nano Lett. 2005, 5, $829-834$

(9) Salem, A. K.; Searson, P. C.; Leong, K. W. Nat. Mater. 2003, 2, 668-671.
(10) Yelin, D.; Oron, D.; Thiberge, S.; Moses, E.; Silberberg, Y. Opt. Express 2003, 11, 1385-1391.

(11) Sönnichsen, C.; Alivisatos, A. P. Nano Lett. 2005, 5, 301-304.

(12) Lindfors, K.; Kalkbrenner, T.; Stoller, P.; Sandoghdar, V. Phvs. Rev. Lett. 2004, 93, 037401-1-037401-4.

(13) Kalkbrenner, T.; Håkanson, U.; Sandoghdar, V. Nano Lett. 2004, 4, 2309-2314

(14) Eah, S.-K.; Jaeger, H. M.; Schererb, N. F.; Wiederrechtc, G. P.; Linc, X.-M. Appl. Phvs. Lett. 2005, 86, 031902-1-031902-3.

(15) Nikoobakht, B.; El-Sayed, M. A. Chem. Mater. 2003, 15, 19571962

(16) Perez-Juste, J.; Marzan, L. M.; Carnie, S.; Chan, D. Y. C.; Mulvaney, P. Adv. Funct. Mater. 2004, 14, 571-579.

(17) Link, S.; Mohamed, M. B.; El-Sayed, M. A. J. Phvs. Chem. B. 1999, 103, 3073-3077.

(18) Novotny, L.; Beversluis, M. R.; Youngworth, K. S.; Brown, T. G. Phys. Rev. Lett. 2001, 86, 5250-5254.

(19) Dorn, R.; Quabis, S.; Leuchs, G. Phvs. Rev. Lett. 2003, 91, 2339011 .

(20) Novotny, L.; Grober, R. B.; Karrai, K. Opt. Lett. 2001, 26, 789791.

(21) Batchelder, J. S.; Taubenblatt, M. A. U.S. Patent 5,037,202, 1991.

(22) Lieb, M. A.; Zavislan, J. M.; Novotny, L. J. Opt. Soc. Am. B. 2004, $21,1210-1215$.

(23) Piwonski, H.; Stupperich, C.; Hartschuh, A.; Sepioł, J.; Meixner, A. J.; Waluk, J. J. Am Chem. Soc. 2005, 127, 5302-5303.

(24) Hui, Qian. Characterization of Individual Nanoparticles by Novel Light-Scattering Microscopic Techniques. Master thesis, University of Siegen, 2005.

NL0603404 